\title{
CARBONO ORGÂNICO E ATRIBUTOS FÍSICOS DO SOLO SOB MANEJO AGROPECUÁRIO SUSTENTÁVEL NA AMAZÔNIA LEGAL
}

\author{
Agust Sales ${ }^{1}$, Arystides Resende Silva², Carlos Alberto Costa Veloso ${ }^{2}$, Eduardo Jorge Maklouf Carvalho ${ }^{2}$, \\ Bárbara Maia Miranda ${ }^{3}$ \\ ${ }^{1}$ Universidade Federal de Viçosa - UFV, Departamento de Engenharia Florestal, Viçosa, MG. E-mail: \\ agustsales@hotmail.com. ²EMBRAPA Amazônia Oriental, Laboratório de Solos, Belém, PA. ${ }^{3}$ Universidade do Estado do \\ Pará - UEPA, Centro de Ciências Naturais e Tecnologia, Belém, PA.
}

\section{RESUMO}

Estudos visando elevar a qualidade dos solos da Amazônia têm crescido na última década em virtude de seu uso inadequado poder limitar a capacidade de sua produção. Na Região Amazônica, pesquisas demonstram que a agropecuária contribui com o aumento de áreas degradadas correlacionadas ao desflorestamento, no entanto, essa prática possui considerável importância na economia. Dessa forma, o objetivo deste trabalho foi avaliar os níveis de densidade, porosidade total, macro e microporosidade, teores de carbono orgânico e quantificar o estoque de carbono nas camadas no perfil do solo em um sistema de ILPF e em sistemas convencionais no município de Paragominas, Pará. Foi utilizado um delineamento inteiramente casualizado, com três repetições, em esquema de parcela subdividida com quatro parcelas (sistemas de manejo do solo) e sete subparcelas (camadas do solo). As parcelas foram compostas por: sistema ILPF-Paricá, lavoura com plantio convencional de milho (PM), pastagem manejada com criação de gado de corte em sistema extensivo (PE) e como testemunha uma floresta secundária (FS). As subparcelas foram as profundidades de amostragem: 0-10, 10-20, 20-30, 30-40, 40-60, 60-80 e 80-100 $\mathrm{cm}$. O sistema de integração ILPF-Paricá, aos cinco anos de cultivo, melhorou as condições físicas de densidade e porosidade do solo, além dos teores e estoques de carbono orgânico nas camadas subsuperficiais. O PM promoveu aumento da densidade do solo e perda de porosidade total e microporosidade em profundidade, mas apresentou teores e estoques de carbono orgânico similar ao sistema de integração Lavoura-Pecuária-Floresta. A pastagem demonstrou teores e estoques de carbono orgânico semelhante à floresta secundária, porém, indicou redução de macroporosidade.

Palavras-chave: agrossilvicultura; ILPF; matéria orgânica do solo; qualidade física do solo; sistema conservacionista.

\section{ORGANIC CARBON AND PHYSICAL ATTRIBUTES OF SOIL UNDER MANAGEMENT SUSTAINABLE AGRICULTURAL IN THE LEGAL AMAZON}

\begin{abstract}
Studies aimed at raising the quality of Amazonian soils have grown in the last decade because their inadequate use may limit the capacity of their production. In the Amazon Region, research shows that agriculture contributes to the increase of degraded areas correlated to deforestation, however, this practice has considerable importance in the economy. Thus, the objective of this work was to evaluate the levels of density, total porosity, macro and microporosity, organic carbon contents and to quantify the carbon stock in the soil profile layers in an ICLF system and in conventional systems in the municipality of Paragominas, Pará. A completely randomized design was used, with three replications, in a plot scheme subdivided with four plots (soil management systems) and seven subplots (soil layers). The plots were composed of: ICLF-Paricá system, conventional tillage maize (TM), pasture managed with cattle breeding in extensive system (PE) and as a secondary forest (FS). The subplots were the sampling depths: 0-10, 10-20, 20-30, 30-40, 40-60, 60-80 and 80-100 cm. The ICLF-Paricá integration system, at five years of cultivation, improved the physical conditions of soil density and porosity, as well as the levels and stocks of organic carbon in the subsurface layers. PM promoted increased soil density and loss of total porosity and microporosity in depth, but presented levels and stocks of organic carbon similar to the integrated crop-
\end{abstract}


livestock-forest (ICLF) system. The pasture showed levels and stocks of organic carbon similar to the secondary forest, but indicated a reduction of macroporosity.

Keywords: agroforestry; ICLF; soil organic matter; soil physical quality; conservationist system.

\section{INTRODUÇÃO}

Estudos visando elevar a qualidade dos solos da Amazônia têm crescido na última década. Em virtude que o uso inadequado deste recurso pode limitar a capacidade em manter ou elevar a produção. Práticas inapropriadas de manejo provocam redução acelerada dos teores de matéria orgânica e elevação dos níveis de compactação do solo, refletindo desfavoravelmente na produtividade das culturas (LINHARES et al., 2016).

Nas últimas cinco décadas, a Amazônia Legal sofreu intensa exploração, fato que proporcionou altos níveis de desmatamento na região. O estado do Pará foi um dos principais responsáveis pelo desflorestamento na região Norte do Brasil, motivado por sua alta produção de grãos, madeira, carne bovina e extração de minérios. Neste contexto, o município de Paragominas foi uma das regiões com maior taxa de desmatamento entre os anos de 1970 e 2000 (IBGE, 2016; INPE, 2016).

$\mathrm{Na}$ Região Amazônica, pesquisas demonstram que a agropecuária contribui com o aumento de áreas degradadas associadas ao desflorestamento, no entanto, essa prática possui considerável importância na economia (DOMINGUES; BERMANN, 2012). Desta maneira, sistemas que visam à conservação dos atributos do solo tornam-se alternativas viáveis para a conservação da qualidade e a sustentabilidade do setor agropecuário.

Manejos dos solos como sistemas de integração Lavoura-Pecuária-Floresta em plantio direto são práticas conservacionistas que vem tornando-se cada vez mais fundamentais para a sustentação da qualidade do solo, pois são capazes de elevar em médio e longo prazo os teores de matéria orgânica (BAYER et al., 2010; LOSS et al., 2011; SILVA, 2016).

A matéria orgânica é formada essencialmente por compostos de carbono e possui implicações no comportamento físico do solo, de maneira a exercer grande influência no processo de restabelecimento estrutural. 0 carbono orgânico está diretamente ligado à qualidade do solo, pois é um agente cimentante da estrutura, atua no tampão do $\mathrm{pH}$, na complexação de elementos e capacidade de troca de cátions, além de aumentar a disponibilidade hídrica no solo (CAMPOS et al., 2016).

Diante da interação do carbono orgânico e os atributos físicos com as atividades de manejo no solo, presume-se que a avaliação dessas variáveis são, portanto, medidas importantes no processo de identificação de práticas mais adequadas com 0 intuito de tornar a agropecuária social e ambientalmente mais sustentável elevando a produtividade, atuando na mitigação de emissão de carbono para atmosfera e reduzindo a pressão sobre as áreas naturais.

O objetivo deste trabalho foi avaliar os níveis de densidade, porosidade total, macro e microporosidade, os teores de carbono orgânico e quantificar o estoque de carbono nas camadas no perfil do solo em um sistema de integração Lavoura-Pecuária-Floresta e em sistemas convencionais no município de Paragominas, Pará.

\section{MATERIAL E MÉTODOS \\ Área experimental}

O estudo foi conduzido na fazenda Vitória, localizada no município de Paragominas, região nordeste do Pará (altitude de 89 metros a $02^{\circ} 57^{\prime} 29,47^{\prime \prime} \mathrm{S}$ de latitude e $47^{\circ} 23^{\prime} 10,37^{\prime \prime} \mathrm{W}$ de longitude). Segundo Koppen, o clima do município é classificado como mesotérmico e úmido tipologia climática Aw. A precipitação média anual é de $1743 \mathrm{~mm}$. A temperatura média anual apresenta variação de $23,3 \circ \mathrm{C}$ a 27,3 으 $\mathrm{e}$ a umidade relativa do ar possui média anual de 81\% (INMET, 2017).

O solo foi classificado como Latossolo Amarelo textura argilosa (EMBRAPA, 2013), sendo os atributos químicos e granulométricos analisados antes da implantação do experimento nas profundidades 0-10, 10-20, 20-30 e 30-50 cm, utilizando a metodologia proposta pela EMBRAPA (2011) (Tabela 1). 
Tabela 1. Atributos químicos e granulométricos da área experimental antes da implantação do experimento.

\begin{tabular}{|c|c|c|c|c|c|c|c|c|c|c|c|}
\hline $\begin{array}{l}\text { Prof. } \\
\text { (cm) }\end{array}$ & $\begin{array}{c}\mathrm{pH} \\
\left(\mathrm{H}_{2} \mathrm{O}\right)\end{array}$ & M.O. & $\mathbf{P}$ & $\mathrm{Ca}$ & Mg & K & Al & $\mathrm{H}+\mathrm{Al}$ & Areia & Silte & Argila \\
\hline & & $\mathrm{g} \mathrm{kg}^{-1}$ & $\mathrm{mg} \mathrm{kg}^{-1}$ & & - $\mathrm{cn}$ & $I_{c} \mathrm{dm}^{-3}$ & & & & $\mathrm{~g} \mathrm{~kg}^{-1}$ & \\
\hline $0-10$ & 5,77 & 24,6 & 5,39 & 3,91 & 1,19 & 0,51 & 0,20 & 3,74 & 56 & 284 & 660 \\
\hline $10-20$ & 5,97 & 19,2 & 9,23 & 4,75 & 1,14 & 0,25 & 0,20 & 2,34 & 43 & 232 & 725 \\
\hline $20-30$ & 5,29 & 15,0 & 1,80 & 1,48 & 0,60 & 0,08 & 0,40 & 3,10 & 39 & 191 & 770 \\
\hline $30-50$ & 5,28 & 14,0 & 1,60 & 1,37 & 0,60 & 0,09 & 0,40 & 2,80 & 35 & 175 & 790 \\
\hline
\end{tabular}

$\mathrm{MO}=$ Matéria Orgânica; $\mathrm{P}=$ Fósforo; $\mathrm{Ca}=$ Cálcio; $\mathrm{Mg}=$ Magnésio; $\mathrm{K}=$ Potássio; $\mathrm{Al}=$ =Alumínio; $\mathrm{H}+\mathrm{Al}=$ Hidrogênio + Alumínio.

\section{Descrição do experimento}

O experimento foi composto por um sistema de integração Lavoura-Pecuária-Floresta cultivado com paricá (ILPF-Paricá), lavoura com plantio convencional de milho (PM), pastagem manejada com criação de gado de corte em sistema extensivo $(\mathrm{PE})$ e como testemunha uma floresta secundária (FS) circunvizinha à área experimental.

Em janeiro de 2009, para implantação do experimento, foram realizadas operações de preparo convencional do solo (aração e gradagem) e correção da acidez, aplicando-se uma dose de calcário (PRNT 90\%) para elevar a saturação por bases a $60 \%$. O corretivo foi incorporado, por ocasião do preparo de solo, sendo metade da dose de calcário aplicada antes da aração e o restante antes da gradagem.

O sistema ILPF-Paricá foi implantado em fevereiro de 2009, área de 4,05 ha com cultivo de culturas anuais (milho e soja) consorciadas com forragem e intercalado com renques de paricá (Schizolobium parahyba var. amazonicum (Huber ex Ducke) Barneby).

O arranjo espacial da espécie florestal no sistema ILPF foi através de renques, cada um com duas linhas, com espaçamento $4 \times 3 \mathrm{~m}$, a distância entre renques foi de $21 \mathrm{~m}$ para o cultivo das culturas anuais e forrageiras, totalizando $24 \%$ da área ocupada pelas faixas dos renques e densidade de 267 árvores ha $^{-1}$.

No plantio do paricá foi aplicado $300 \mathrm{~g}$ de fosfato Arad e $100 \mathrm{~g}$ de super fosfato simples por cova. A adubação de cobertura foi realizada em maio de 2009, após o coroamento das mudas, com $60 \mathrm{~g}$ de ureia e $40 \mathrm{~g}$ de $\mathrm{KCl}$ por planta.

No ano de 2009 foi semeado o milho BRS 1030, em 2010 a soja (cultivar Sambaiba) e nos anos de 2011 e 2012 o milho BRS 1055, todos semeados em espaçamento de $0,6 \mathrm{~m}$ com adubação de base de $330 \mathrm{~kg} \mathrm{ha}^{-1}$ de NPK na formulação 10-28-20 e com adubação de cobertura realizada após 30 dias de plantio com aplicação de $200 \mathrm{~kg} \mathrm{ha}^{-1}$ de Ureia $+\mathrm{KCl}, 2: 1$. Em 2009, 2010 e 2011, na adubação de cobertura foi semeada a forragem Brachiaria ruziziensis $(20 \mathrm{~kg}$ $\mathrm{ha}^{-1}, 72 \% \mathrm{VC}$ ) entre as linhas de plantio do milho para produção de feno no semestre seguinte.

Em 2012, na adubação de cobertura foi semeado a Brachiaria brizantha cv. piatã $(20 \mathrm{~kg}$ $\mathrm{ha}^{-1}, 72 \% \mathrm{CV}$ ) para pastejo direto no semestre seguinte, a qual permaneceu até o final da avaliação do estudo. Todos os cultivos foram realizados de acordo com as recomendações técnicas para as culturas.

A área do PM (plantio convencional de milho), entre 2009 e 2012, foi manejado com cultivo alternado de milho e soja em 5 ha, com técnicas tradicionais de preparo do solo (aração e gradagem) e controle fitossanitário. Em fevereiro de 2014, foi realizada a semeadura do milho BRS 1040 em linhas no espaçamento de 0,60 m, após aplicação de glifosato, com adubação de base de $330 \mathrm{~kg} \mathrm{ha}^{-1} \mathrm{da}$ formulação 10-28-20. Em março e maio de 2012, foram realizadas adubações de cobertura com $200 \mathrm{~kg} \mathrm{ha}^{-1}$ (Ureia $+\mathrm{KCl}, 2: 1$ ) e 180 $\mathrm{kg}$ (Ureia $+\mathrm{KCl}, 2: 1$ ), respectivamente.

A área da pastagem manejada com criação de gado de corte em sistema extensivo (PE) foi implantada em 1969 após remoção da vegetação nativa manejada com Panicum maximum (cultivar colonião) até 1975 , renovação de pastagem com plantio da Brachiaria humidicola até 1999, sendo substituída pela semeadura de Brachiaria brizantha (cultivar marandu) e recebendo correção/adubação do solo periodicamente.

A floresta secundária (FS) utilizada como referência no momento da coleta apresentava 38 anos em processo de regeneração natural. Até 1969, a área apresentava vegetação nativa, sendo removida para formação de pastagem com 
exploração de gado de corte em sistema extensivo com cultivo de Panicum maximum (cultivar colonião) até 1975, totalizando sete anos de pastejo convencional. Desde 1976, a área é mantida como área de preservação permanente.

Foi utilizado um delineamento inteiramente casualizado, com três repetições, em esquema de parcela subdividida com quatro parcelas (sistemas de manejo do solo) e sete subparcelas (camadas do solo). As parcelas foram compostas por: sistema ILPF-Paricá (amostrado a $5 \mathrm{~m}$ do renque florestal), PM, PE e FS. As subparcelas foram as profundidades de amostragem: 0-10, 10-20, 20-30, 30-40, 40-60, $60-80$ e $80-100 \mathrm{~cm}$.

Em 2014 foram realizadas análises químicas do solo seguindo a metodologia proposta por EMBRAPA (2011) (Tabela 2).

Tabela 2. Características químicas do solo dos tratamentos.

\begin{tabular}{|c|c|c|c|c|c|c|c|c|c|}
\hline Tratamentos* & $\begin{array}{l}\text { Prof. } \\
\text { (cm) }\end{array}$ & $\begin{array}{c}\mathrm{pH} \\
\left(\mathrm{H}_{2} \mathrm{O}\right)\end{array}$ & $\begin{array}{l}\text { M.O. } \\
\left(\mathrm{g} \mathrm{kg}^{-1}\right)\end{array}$ & $\begin{array}{c}P \\
\left(\mathrm{mg} \mathrm{dm}^{-3}\right)\end{array}$ & $\mathrm{Ca}$ & $\mathrm{Ca}+\mathrm{Mg}$ & K & Al & $\begin{array}{l}\mathrm{H}+ \\
\mathrm{Al}\end{array}$ \\
\hline & & & & & & $\mathrm{cn}$ & $\mathrm{ol}_{\mathrm{c}} \mathrm{dm}^{-1}$ & & \\
\hline & $0-10$ & 4,63 & 32,3 & 6,0 & 1,33 & 1,90 & 0,18 & 0,80 & 6,00 \\
\hline & $10-20$ & 4,57 & 22,7 & 2,0 & 0,93 & 1,33 & 0,07 & 0,83 & 5,40 \\
\hline & $20-30$ & 4,50 & 17,0 & 1,0 & 0,43 & 0,67 & 0,03 & 0,97 & 4,63 \\
\hline \multirow[t]{7}{*}{ ILPF-Paricá } & $30-40$ & 4,60 & 11,3 & 1,0 & 0,37 & 0,57 & 0,03 & 0,83 & 4,03 \\
\hline & $40-60$ & 4,90 & 8,3 & 1,0 & 0,37 & 0,57 & 0,02 & 0,43 & 3,07 \\
\hline & $60-80$ & 5,30 & 5,7 & 1,0 & 0,50 & 0,73 & 0,03 & 0,17 & 2,33 \\
\hline & $80-100$ & 5,30 & 3,7 & 1,0 & 0,43 & 0,67 & 0,02 & 0,13 & 2,17 \\
\hline & $0-10$ & 4,47 & 35,3 & 17,0 & 1,93 & 2,53 & 0,61 & 0,90 & 6,53 \\
\hline & $10-20$ & 4,17 & 26,7 & 4,0 & 0,80 & 1,17 & 0,16 & 1,40 & 8,13 \\
\hline & $20-30$ & 4,30 & 19,7 & 2,4 & 0,47 & 0,70 & 0,11 & 1,07 & 6,00 \\
\hline \multirow[t]{7}{*}{ PM } & $30-40$ & 4,50 & 14,3 & 1,0 & 0,50 & 0,73 & 0,10 & 0,87 & 4,53 \\
\hline & $40-60$ & 4,63 & 10,0 & 1,4 & 0,37 & 0,53 & 0,09 & 0,73 & 3,93 \\
\hline & $60-80$ & 5,07 & 7,0 & 1,0 & 0,40 & 0,60 & 0,07 & 0,33 & 2,67 \\
\hline & $80-100$ & 5,37 & 5,0 & 1,0 & 0,53 & 0,80 & 0,06 & 0,13 & 2,13 \\
\hline & $0-10$ & 4,03 & 61,3 & 11,4 & 3,40 & 4,77 & 0,40 & 0,13 & 3,73 \\
\hline & $10-20$ & 3,98 & 26 & 2,0 & 1,50 & 2,13 & 0,18 & 0,17 & 3,17 \\
\hline & $20-30$ & 4,00 & 18,3 & 1,0 & 1,07 & 1,57 & 0,12 & 0,17 & 2,67 \\
\hline \multirow[t]{7}{*}{ PE } & $30-40$ & 4,15 & 14,0 & 1,0 & 0,97 & 1,37 & 0,05 & 0,11 & 2,17 \\
\hline & $40-60$ & 4,43 & 10,0 & 1,0 & 0,83 & 1,23 & 0,03 & 0,11 & 1,93 \\
\hline & $60-80$ & 4,58 & 7,0 & 1,0 & 0,70 & 1,07 & 0,02 & 0,09 & 1,73 \\
\hline & $80-100$ & 4,38 & 5,0 & 1,0 & 0,67 & 1,10 & 0,02 & 0,07 & 1,67 \\
\hline & $0-10$ & 4,97 & 56,0 & 3,0 & 4,73 & 6,07 & 0,10 & 0,43 & 5,00 \\
\hline & $10-20$ & 5,07 & 31,0 & 1,67 & 1,60 & 2,40 & 0,05 & 0,27 & 4,00 \\
\hline & $20-30$ & 5,10 & 18,0 & 1,0 & 0,97 & 1,47 & 0,04 & 0,27 & 3,27 \\
\hline \multirow[t]{4}{*}{ FS } & $30-40$ & 5,13 & 12,3 & 1,0 & 0,77 & 1,17 & 0,03 & 0,20 & 2,63 \\
\hline & $40-60$ & 5,17 & 9,3 & 1,0 & 0,63 & 0,93 & 0,02 & 0,20 & 2,57 \\
\hline & $60-80$ & 5,27 & 5,7 & 1,0 & 0,43 & 0,63 & 0,02 & 0,17 & 2,30 \\
\hline & $80-100$ & 5,50 & 3,7 & 1,0 & 0,37 & 0,53 & 0,03 & 0,07 & 1,90 \\
\hline
\end{tabular}

ILPF-Paricá = Sistema de integração Lavoura-Pecuária-Floresta cultivado com paricá; PM = Plantio convencional de milho; PE = Pastagem manejada com criação de gado de corte em sistema extensivo; FS = Floresta secundária.

\section{Amostragem do solo}

De acordo com Araújo e Monteiro (2007), a maior parcela da atividade biológica no solo está concentrada na profundidade de $0-30 \mathrm{~cm}$. Entretanto, é importante ressaltar que o solo deve ser analisado em maior profundidade, visto que este é um dos principais depósitos de carbono no ecossistema terrestre (LAL, 2005). Por essa razão, o presente trabalho avaliou os estoques de carbono em camadas, separadamente, até $100 \mathrm{~cm}$ de profundidade. 
A coleta de solo foi realizada em março de 2014 através da abertura de uma trincheira em cada sistema, ILPF-Paricá (a $5 \mathrm{~m}$ de distância dos renques florestais), PM, PE e FS. A amostragem ocorreu em três paredes de cada trincheira, obtendo replicações. Foram coletadas amostras com estruturas deformadas e indeformadas utilizando o método descrito por Blake e Hartge (1986). As amostras foram identificadas e as localizações das trincheiras georreferenciadas.

\section{Análises de solo}

As análises de carbono orgânico, densidade, porosidade total, macro e microporosidade do solo foram realizadas utilizando a metodologia descrita por EMBRAPA (2011).

\section{Estoque de carbono}

Após a determinação da Ds e do teor de COS foi possível calcular o estoque de carbono do solo pelo método da camada proposto por Carvalho et al. (2009). O cálculo do estoque de cada camada amostrada foi realizado pela equação (1):

$$
E C=\frac{C O S \times D s \times\left(\frac{D r e f}{D s} \times e\right)}{10}
$$

$\mathrm{EC}=$ estoque de carbono $\left(\mathrm{Mg} \mathrm{ha}^{-1}\right)$

COS = teor de carbono orgânico do solo $\left(\mathrm{g} \mathrm{kg}^{-1}\right)$

Ds $=$ densidade do solo $\left(\mathrm{kg} \mathrm{dm}^{-3}\right)$

Dref $=$ densidade do solo para profundidade amostrada na área de referência $\left(\mathrm{kg} \mathrm{dm}^{-3}\right)$

$\mathrm{e}=$ espessura da camada amostrada $(\mathrm{cm})$

\section{Análises estatísticas}

Os resultados obtidos em cada tratamento foram comparados entre as camadas avaliadas e entre cada tratamento e submetidos à análise de variância através do programa estatístico SISVAR ${ }^{\circ}$ e quando significativo às médias foram comparadas pelo teste de Tukey $\mathrm{p}<0,05$ (FERREIRA, 2011).

\section{RESULTADOS E DISCUSSÃO}

Os teores de COS foram influenciados pela profundidade do solo. Os maiores teores de COS foram observados na camada mais superficial de todos os tratamentos, indicando redução em profundidade (Tabela 3 ).

Tabela 3. Teor de Carbono orgânico do solo (COS).

\begin{tabular}{|c|c|c|c|c|c|c|}
\hline \multirow{2}{*}{ Variável } & \multirow{2}{*}{$\begin{array}{l}\text { Prof. } \\
(\mathrm{cm})\end{array}$} & \multicolumn{5}{|c|}{ Tratamentos* } \\
\hline & & ILPF-Paricá & PM & $\mathrm{PE}$ & FS & $C V(\%)^{2}$ \\
\hline \multirow{8}{*}{$\begin{array}{c}\cos \\
\left(\mathrm{g} \mathrm{kg}^{-1}\right)\end{array}$} & $0-10$ & $19,75 B a$ & $20,49 B a$ & $35,57 \mathrm{Aa}$ & $32,48 \mathrm{Aa}$ & 9,36 \\
\hline & $10-20$ & $15,87 \mathrm{Ab}$ & $15,47 \mathrm{Ab}$ & $15,08 \mathrm{Ab}$ & $17,98 \mathrm{Ab}$ & 8,34 \\
\hline & $20-30$ & $10,56 \mathrm{Ac}$ & $11,41 \mathrm{Ac}$ & $10,63 A c$ & $10,40 A c$ & 12,15 \\
\hline & $30-40$ & 7,57Ad & $8,31 \mathrm{Ad}$ & $8,12 A d$ & 7,15Acd & 10,88 \\
\hline & $40-60$ & 5,83Ade & $5,80 \mathrm{Ae}$ & 5,80Ade & $5,41 \mathrm{Ad}$ & 6,85 \\
\hline & $60-80$ & 3,89Aef & 3,94Aef & 4,06Aef & $3,92 \mathrm{Ae}$ & 12,89 \\
\hline & $80-100$ & $2,33 \mathrm{Af}$ & $2,80 \mathrm{Af}$ & $2,90 \mathrm{Af}$ & $2,45 \mathrm{Ae}$ & 11,43 \\
\hline & $C V(\%)^{1}$ & 9,07 & 6,47 & 7,15 & 8,23 & - \\
\hline
\end{tabular}

Médias seguidas da mesma letra maiúscula na linha e minúscula na coluna não diferem entre si pelo teste de Tukey $(p<0,05)$.

${ }^{1} \mathrm{CV}(\%)$ coeficiente de variação referente aos tratamentos (parcelas).

${ }^{2} \mathrm{CV}(\%)$ coeficiente de variação referente às camadas (subparcelas).

Houve comportamento semelhante em relação aos teores de COS: maior valor na camada $0-10 \mathrm{~cm}$, valores intermediários nas camadas $10-20,20-30,30-40,40-60 \mathrm{~cm}$ e os menores valores nas camadas $60-80$ e $80-100 \mathrm{~cm}$ (Tabela 3).

Resultados que reforçam os obtidos por Silva et al. (2016), que avaliaram o teor de COS sob influência de árvores de eucalipto e diferentes sistemas de manejo do solo, relataram diminuição de COS conforme aumentou a profundidade.

Este fato é corroborado por outros autores (COSTA JR et al., 2012; ARRUDA et al., 2015; GUAN et al., 2015), ao relatarem que o conteúdo de COS do solo é maior próximo da superfície, por causa dos aportes de matéria orgânica ocorridos via cobertura vegetal.

Ao comparar os tratamentos, na camada 0-10 cm houve diferença significativa, o FS e PE 
apresentaram os maiores valores de COS, não diferindo entre si. Nas demais profundidades, os sistemas de manejo e uso do solo não diferiram entre si (Tabela 3).

Resultados semelhantes foram obtidos por Linhares et al. (2016), ao avaliarem atributos do solo em sistemas de cultivo distintos, encontraram valores superiores de $\operatorname{COS}$ na profundidade de $0-10 \mathrm{~cm}$ em floresta nativa e pastagem, quando relacionados à sistemas agroflorestais e agricultura convencional. Isso pode ser explicado devido a ausência de revolvimento do solo e remoção de serrapilheira na floresta e no pasto, da incorporação dos resíduos vegetais advindos do roço de ervas daninhas e a possível presença de carbono recalcitrante na forma de carvão gerado por meio do tradicional método de queima da floresta primária para implantação do pasto.

No ILPF-Paricá o teor de COS na camada 0-10 cm foi semelhante ao PM e inferior à FS (Tabela 3), esse fato pode ser explicado devido o sistema ser considerado jovem apresentando, no momento da coleta de solo, cinco anos de implantação, além disso, foi implantando em área com histórico recente de pastagem degradada e para instalação do sistema o solo foi revolvido, corrigido e adubado, condições que aceleraram o processo de decomposição da MOS, propiciando ainda mais a atenuação de COS.

Ressalta-se que a camada superficial do solo é considerada como a mais sensível ao manejo e está associada com a entrada de compostos no solo mais facilmente decomponíveis encontrados nos resíduos das culturas (SOUZA et al., 2009).

Pesquisas recentes (LIMA et al., 2011; IWATA et al., 2013; LINHARES et al., 2016) revelam que sistemas agroflorestais, oriundos de pastagens degradadas, podem necessitar de maior período de adoção para apresentar níveis de COS similares ou superiores à vegetação nativa, sendo influenciado pela quantidade fornecida de material orgânico como restos culturais, excreções radiculares, serrapilheira, entre outros.

Estudo conduzido por Linhares et al. (2016), mostrou que a implantação de sistemas agroflorestais por período superior a dez anos foi eficiente na recuperação do $\mathrm{COS}$, comparando-se à floresta nativa. Guareschi et al. (2012), ressaltam que a manutenção de fitomassa na Região Amazônica é dificultada em razão da elevada temperatura e umidade, fatores que aceleram o processo de decomposição e impedem o acúmulo de carbono no solo. Sendo assim, é recomendado o uso de sistemas de manejo do solo com rotações de culturas de maior aporte de fitomassa, para que após os processos de decomposição/mineralização, ocorra incorporação de carbono no solo.

Os elevados teores de COS na camada superficial nos tratamentos FS e PE pode ser devido à deposição de material orgânico vegetal (fitomassa), de resíduos orgânicos dos animais (urina e fezes), rizodeposição das plantas e manutenção da estrutura do solo (SILVA et al., 2011), fatores que estão ligados à ausência de revolvimento do solo e remoção do material orgânico, condição que favorece maior atividade dos organismos responsáveis pela transformação do tecido vegetal em compostos orgânicos, dentre outros elementos fundamentais para o agroecossistema (BARTZ et al., 2014).

A densidade do solo (Ds) demonstrou diferença significativa entre as profundidades em todos os tratamentos, sendo demonstrado um aumento de Ds em profundidade no ILPF-Paricá, $\mathrm{PM}$ e FS quando comparado a camada $0-10 \mathrm{~cm}$ com as demais profundidades, e no PE a partir da camada 60-80 cm (Tabela 4). 
Tabela 4. Densidade do solo (Ds).

\begin{tabular}{ccccccc}
\hline \multirow{2}{*}{ Variável } & $\begin{array}{c}\text { Prof. } \\
\text { (cm) }\end{array}$ & ILPF-Paricá & PM & PE & FS & CV (\%) \\
\cline { 3 - 6 } & $0-10$ & $1,05 \mathrm{Ba}$ & $1,10 \mathrm{Ca}$ & $1,21 \mathrm{Da}$ & $0,89 \mathrm{Aa}$ & 4,03 \\
& $10-20$ & $1,16 \mathrm{Aab}$ & $1,41 \mathrm{Cb}$ & $1,20 \mathrm{Ba}$ & $1,15 \mathrm{Ab}$ & 1,42 \\
& $20-30$ & $1,25 \mathrm{Ab}$ & $1,42 \mathrm{Bb}$ & $1,21 \mathrm{Aa}$ & $1,19 \mathrm{Ab}$ & 3,02 \\
Ds & $30-40$ & $1,21 \mathrm{Ab}$ & $1,44 \mathrm{Bb}$ & $1,20 \mathrm{Aa}$ & $1,13 \mathrm{Ab}$ & 2,59 \\
$\left(\mathbf{k g ~ d m}^{-3}\right)$ & $40-60$ & $1,22 \mathrm{Ab}$ & $1,33 \mathrm{Bb}$ & $1,22 \mathrm{Aa}$ & $1,18 \mathrm{Ab}$ & 5,20 \\
& $60-80$ & $1,26 \mathrm{Ab}$ & $1,36 \mathrm{Bb}$ & $1,25 \mathrm{Ab}$ & $1,19 \mathrm{Ab}$ & 3,06 \\
& $80-100$ & $1,24 \mathrm{Ab}$ & $1,42 \mathrm{Bb}$ & $1,26 \mathrm{Ab}$ & $1,18 \mathrm{Ab}$ & 2,92 \\
& $C V(\%)^{1}$ & 4,18 & 3,27 & 2,13 & 3,29 & - \\
\hline
\end{tabular}

* Médias seguidas da mesma letra maiúscula na linha e minúscula na coluna não diferem entre si pelo teste de Tukey $(p<0,05)$.

${ }^{1} \mathrm{CV}(\%)$ coeficiente de variação referente aos tratamentos (parcelas).

${ }^{2} \mathrm{CV}(\%)$ coeficiente de variação referente às camadas (subparcelas).

Resultados similares foram obtidos por Rossetti e Centurion (2015), no trabalho em que quantificaram os atributos físicos de um Latossolo em uma cronossequência sob diferentes manejos, descreveram aumento de Ds em profundidade. Resultados também observado por Torres et al. (2015), ao avaliarem as alterações nos atributos físicos do solo após doze anos de plantio direto.

Na comparação de Ds dos tratamentos entre cada profundidade, a FS indicou os menores valores, diferiu do ILPF-Paricá apenas na camada 0-10 cm, diferiu do PE de 0-10 e 10-20 $\mathrm{cm}$ e quando comparada ao PM, diferiu em todas as camadas (Tabela 4).

Ao considerar apenas os valores de Ds, tais resultados indicam que o ILPF-Paricá, PM e FS (Tabela 6) não danificaram este atributo a ponto de torná-lo superior ao nível crítico de 1,30 a 1,40 $\mathrm{kg} \mathrm{m}^{-3}$ para solos argilosos (REICHERT et al., 2009), pois Silva et al. (2011), relataram que quando for identificado Ds superior a $1,30 \mathrm{~kg} \mathrm{~m}^{-3}$ poderá ocorrer restrições ao desenvolvimento radicular da maioria das plantas. Foi observado no PM valores de Ds (Tabela 4) acima do nível crítico mínimo $\left(1,30 \mathrm{~kg} \mathrm{~m}^{-3}\right)$, que pode ser atribuído à ausência de planta de cobertura e à camada compactada resultante do preparo de solo anteriores com aração e gradagem (OLIVEIRA et al., 2013).

Segundo Iwata et al. (2013), os sistemas agroflorestais são sistemas mais conservacionistas se comparados à agricultura convencional e à pecuária extensiva, isso pode ser notado em decorrência do aumento de Ds nas primeiras camadas do tratamento PE e em todas as camadas do PM (Tabela 4).
Os valores de Ds nas camadas superficiais do PE (Tabela 4) podem ser explicados pela formação do "pé de vaca" que consiste na compactação do solo em razão do aumento de Ds nas camadas superficiais em áreas de pastagens devido ao impacto negativo do pisoteio animal em condições de alta umidade e à má distribuição espacial (lotação) dos animais (UA ha $\left.{ }^{1}\right)$ (KAISER, 2010; LINHARES et al., 2016).

No PM, os altos valores de Ds a partir da camada $10-20 \mathrm{~cm}$ (Tabela 4) provavelmente ocorreram devido o "pé de arado", que é resultado das camadas de solo compactadas que se desenvolve abaixo da camada que é anualmente arada e gradeada. Como estes implementos agrícolas não atingem essas camadas, elas permanecem ano após ano e vão sendo compactadas pela perda de sua porosidade através da colmatação e pelas partículas argilosas que são remobilizadas da camada superior (KAISER, 2010; MEDEIROS, 2016).

Esse aumento de Ds nas camadas subsuperficiais provoca restrições à penetração das raízes dos vegetais prejudicando seu desenvolvimento, além disso, sua menor porosidade impede a infiltração da água, o que leva ao aparecimento de erosão em ravinas e à degradação do solo (SOARES et al., 2016).

$\mathrm{O}$ uso do plantio direto, aliado a uma adequada calagem do solo, são práticas que devem ser adotadas para impossibilitar o surgimento destas camadas compactadas. Solos manejados sob o sistema do plantio direto são técnicas consideradas menos invasivas quanto à estrutura do solo, mais preservacionistas e não 
desenvolvem o "pé de arado" e "pé de vaca" (BETIOLI JUNIOR et al., 2012).

Os resultados de Ds no ILPF-Paricá são justificados pelo fato de ter sido inserido no sistema a forragem Brachiaria ruziziensis como planta de cobertura, promovendo formação de matéria orgânica que melhora a estruturação do solo, pois contribui na cimentação e a estabilização dos agregados do solo (SOARES et al., 2016), além de suavizar o impacto negativo proporcionado pelo pisoteio animal e distribuir uniformemente $\mathrm{o}$ peso das máquinas $\mathrm{e}$ implementos agrícolas (LINHARES et al., 2016), enquanto no PM não houve introdução da forragem, além do mais, o tráfego de máquinas e implementos agrícolas na área foi intenso, devido a necessidade de limpeza, aração e gradagem, refletindo em aumento da Ds nas camadas subsuperficiais.

A porosidade total do solo (Pt), indicou diferença significativa nos tratamentos ILPFParicá, PM e FS com perda de porosidade em profundidade. Em geral, os tratamentos quando comparados à FS, o ILPF-Paricá apresentou perda de porosidade nas camadas $60-80$ e $80-100 \mathrm{~cm}$, o PE somente na camada $0-10 \mathrm{~cm}$ e o PM indicou perda de porosidade em todas as profundidades (Tabela 5).

Tabela 5. Porosidade total (Pt), Macroporosidade (MAC) e Microporosidade (MIC).

\begin{tabular}{|c|c|c|c|c|c|c|}
\hline \multirow{2}{*}{ Variável } & \multirow{2}{*}{$\begin{array}{l}\text { Prof. } \\
\text { (cm) }\end{array}$} & \multicolumn{5}{|c|}{ Tratamentos $^{*}$} \\
\hline & & ILPF-Paricá & PM & PE & FS & $C V(\%)^{2}$ \\
\hline \multirow{8}{*}{$\begin{array}{c}\mathrm{Pt} \\
\left(\mathrm{m}^{3} \mathrm{~m}^{-3}\right)\end{array}$} & $0-10$ & $0,55 \mathrm{ABa}$ & $0,51 \mathrm{Ba}$ & $0,47 \mathrm{Ca}$ & $0,58 \mathrm{Aa}$ & 3,0 \\
\hline & $10-20$ & $0,49 A b$ & $0,34 \mathrm{Bb}$ & $0,47 \mathrm{Aa}$ & $0,50 \mathrm{Abc}$ & 2,72 \\
\hline & $20-30$ & $0,48 \mathrm{Ab}$ & 0,38Bab & $0,48 \mathrm{Aa}$ & $0,49 A c$ & 6,94 \\
\hline & $30-40$ & $0,49 A b$ & 0,32Bb & 0,49Aa & $0,51 \mathrm{Abc}$ & 7,26 \\
\hline & $40-60$ & $0,48 \mathrm{Ab}$ & $0,38 \mathrm{Bab}$ & $0,48 \mathrm{Aa}$ & $0,53 \mathrm{Ab}$ & 7,07 \\
\hline & $60-80$ & $0,39 \mathrm{BC}$ & $0,40 \mathrm{Bab}$ & $0,48 \mathrm{Aa}$ & $0,52 \mathrm{Abc}$ & 8,98 \\
\hline & $80-100$ & $0,37 \mathrm{BC}$ & $0,35 \mathrm{Bb}$ & $0,48 \mathrm{Aa}$ & $0,52 \mathrm{Abc}$ & 8,80 \\
\hline & $C V(\%)^{1}$ & 7,03 & 13,07 & 2,23 & 2,06 & - \\
\hline \multirow{8}{*}{$\begin{array}{c}\text { MAC } \\
\left(\mathrm{m}^{3} \mathrm{~m}^{-3}\right)\end{array}$} & $0-10$ & $0,19 A a$ & 0,19Aa & 0,07Bab & $0,13 \mathrm{ABab}$ & 11,30 \\
\hline & $10-20$ & $0,13 \mathrm{ABa}$ & $0,15 \mathrm{Aa}$ & $0,09 \mathrm{Ca}$ & $0,12 \mathrm{BCab}$ & 5,58 \\
\hline & $20-30$ & $0,10 \mathrm{Ba}$ & $0,18 \mathrm{Aa}$ & 0,07Cab & $0,12 \mathrm{Bab}$ & 12,28 \\
\hline & $30-40$ & $0,12 A a$ & $0,17 A a$ & 0,06Bab & 0,13Aab & 13,66 \\
\hline & $40-60$ & $0,11 \mathrm{Ba}$ & $0,18 \mathrm{Aa}$ & $0,05 \mathrm{Cb}$ & 0,14Ba & 10,76 \\
\hline & $60-80$ & $0,13 \mathrm{Aa}$ & $0,17 \mathrm{Aa}$ & $0,03 \mathrm{Cb}$ & $0,10 \mathrm{Bb}$ & 15,79 \\
\hline & $80-100$ & $0,11 \mathrm{Aa}$ & $0,11 \mathrm{Aa}$ & $0,04 \mathrm{Bb}$ & $0,10 A b$ & 13,28 \\
\hline & $C V(\%)^{1}$ & 12,57 & 11,97 & 12,52 & 12,47 & - \\
\hline \multirow{8}{*}{$\begin{array}{c}\text { MIC } \\
\left(\mathrm{m}^{3} \mathrm{~m}^{-3}\right)\end{array}$} & $0-10$ & $0,36 \mathrm{ABa}$ & 0,33Ba & $0,39 A B b c$ & $0,44 A a$ & 8,62 \\
\hline & $10-20$ & $0,36 \mathrm{Ba}$ & $0,19 \mathrm{Cb}$ & $0,38 \mathrm{ABC}$ & $0,39 A c$ & 3,08 \\
\hline & $20-30$ & $0,38 \mathrm{Aa}$ & $0,20 \mathrm{Bab}$ & $0,41 A a b$ & $0,38 \mathrm{Ac}$ & 8,41 \\
\hline & $30-40$ & $0,37 \mathrm{Ba}$ & $0,15 \mathrm{Cb}$ & 0,43Aab & $0,38 \mathrm{ABc}$ & 6,87 \\
\hline & $40-60$ & $0,36 \mathrm{Ba}$ & $0,20 \mathrm{Cab}$ & $0,43 \mathrm{Aab}$ & $0,39 A B b c$ & 7,34 \\
\hline & $60-80$ & $0,26 \mathrm{Bb}$ & 0,22Bab & $0,44 \mathrm{Aa}$ & 0,42Aab & 12,09 \\
\hline & $80-100$ & $0,26 \mathrm{Bb}$ & 0,24Bab & 0,44Aab & 0,42Aab & 10,49 \\
\hline & $C V(\%)^{1}$ & 8,57 & 11,51 & 3,89 & 3,46 & - \\
\hline
\end{tabular}

Médias seguidas da mesma letra maiúscula na linha e minúscula na coluna não diferem entre si pelo teste de Tukey $(\mathrm{p}<0,05)$.

${ }^{1} \mathrm{CV}(\%)$ coeficiente de variação referente aos tratamentos (parcelas).

${ }^{2} \mathrm{CV}(\%)$ coeficiente de variação referente às camadas (subparcelas).

A proximidade de valores de $\mathrm{Pt}$ dos tratamentos ILPF-Paricá e PE com o FS e a manutenção da porosidade do solo, ocorreu, possivelmente, por não ter efetuado nestes sistemas o revolvimento contínuo do solo, assim como em razão do sistema radicular das forrageiras e de invertebrados edáficos, que podem ter favorecido a melhoria da sua estruturação física (SANTOS et al., 2011). 
Evidencia-se a essencial importância dos resíduos vegetais na estruturação do solo devido promover maior formação de matéria orgânica e estabilidade dos agregados do solo em virtude à intensa atividade biológica resultando em aeração e infiltração de água no sistema, permitindo o desenvolvimento radicular das culturas (SILVA et al., 2016).

A Pt é representada pelos espaços do solo ocupados por água e/ou ar. A Ds tem relação direta com sua $\mathrm{Pt}$, onde à medida que ocorre aumento de Ds reduz a porosidade, caso encontrado neste trabalho, principalmente no tratamento PM como consequência amplia a resistência do solo à penetração e limita a velocidade de infiltração de água, variáveis que quando modificadas comprometem ou dificultam o desenvolvimento vegetativo e a produção (CRUZ et al., 2014).

A macroporosidade do solo (MAC) apresentou distinção entre as profundidades apenas nos tratamentos FS e PE indicando maiores valores de MAC nas camadas superficiais (Tabela 5). De acordo com Vezzani e Mielniczuk (2011) e Silva et al. (2016), maiores teores de MAC nas camadas superficiais refletem ação positiva da matéria orgânica na estruturação de solos.

Em relação aos tratamentos em cada profundidade, O PE apresentou os menores valores de MAC, diferiu dos demais tratamentos em todas as profundidades (Tabela 5), e foi o único tratamento a apresentar valores de MAC abaixo do nível crítico $0,10 \mathrm{~m}^{3} \mathrm{~m}^{-3}$, valor mínimo de MAC adequado para as trocas gasosas e líquidas entre o ambiente externo e o solo, considerado crítico para o desenvolvimento radicular da maioria das espécies vegetais (BAVER et al., 1972; REICHERT et al., 2007).

Os baixos valores de MAC no tratamento PE podem ser início de deformação dos macroporos do solo ou fechamento dos poros por parte da fração silte e colmatação, promovendo provável compactação do solo (KAISER, 2010; MEDEIROS, 2016).

Em contrapartida, no tratamento PM os altos valores de MAC (Tabela 5) podem estar relacionados ao preparo do solo que foi realizado com aração e gradagem, proporcionando temporariamente alto volume de macroporos.

Embora os macroporos sejam considerados mais suscetíveis a sofrer com perturbações do que os microporos, tais alterações são baixas em sistemas perenes de produção, fazendo com que haja uma ciclagem mais lenta dos macroporos e, dessa forma, o COS existente nesse fragmento torna-se mais estabilizado ao longo do tempo. Ainda, a inexistência de preparo e revolvimento do solo por implementos agrícolas auxilia para a não deterioração dos agentes ligantes, propiciando a manutenção e conservação dessa porção do solo (BETIOLI JUNIOR et al., 2012).

$\mathrm{Na}$ avaliação da microporosidade do solo (MIC) dos tratamentos entre as profundidas, houve diferença significativa entre as camadas, o PE e FS apresentaram aumento de MIC em profundidade, já os tratamentos ILPF-Paricá e PM indicaram maiores valores de MIC nas camadas superficiais com redução em profundidade. Ao comparar os tratamentos em cada profundidade, foi observado no PE e FS os maiores valores de MIC, não diferiram do ILPF-Paricá nas camadas 60-80 e 80-100 cm, diferindo do PM em todas as profundidades amostradas (Tabela 5).

Os resultados de MIC foram similares aos obtidos por Santos et al. (2011) e Silva et al. (2016), onde encontraram maiores valores de MIC quando comparados com MAC, em área de vegetação natural e em áreas sob sistemas consorciados.

De acordo com Kiehl (1979) e Baver et al. (1972), o solo adequado para a produção agropecuária deve apresentar porosidade total média de $0,50 \mathrm{~m}^{3} \mathrm{~m}^{-3}$ com uma distribuição percentual média de $34 \%$ para macroporos e $66 \%$ para microporos, para que haja desenvolvimento satisfatório de sistemas radiculares. Considerando os valores indicados pelos autores, apenas o ILPF-Paricá e FS enquadraram-se como ideal dentro da média dos limites determinados (Tabela 5).

Os altos volumes de MIC no tratamento PE podem estar associados ao adensamento promovido pelo pisoteio animal. Isso provoca desarranjo das partículas do solo, comprometendo a porosidade, a infiltração e a redistribuição de água no solo pelo entupimento dos macroporos pelas partículas finas do solo e, como consequência, aumenta a compactação (LINHARES et al., 2016).

Segundo Oliveira et al. (1996), a expressiva compactação nas camadas subsuperficiais do solo é atribuída à disposição de partículas, em consequência do entupimento dos poros pelas partículas mais finas (silte) e dos ciclos de umedecimento e secagem do solo. 
A MIC está associada à textura do solo que é uma característica pedológica e pode sofrer alterações pelo uso, no entanto, a influência nessa característica é pequena (COSTA et al., 2015; GRECO et al., 2012; STEFANOSKI et al., 2013). Vendruscolo et al. (2011), relataram para solos argilosos maior proporção de microporos do que macroporos, fato encontrado neste trabalho.
Em relação ao estoque de carbono no solo (EC), os tratamentos apresentaram os maiores EC na camada superficial com redução em profundidade. Os tratamentos $F S$ e PE apresentaram os maiores EC, em comparação aos demais na camada de $0-10 \mathrm{~cm}$. Na profundidade $10-20 \mathrm{~cm}$ o FS demonstrou maiores EC, nas camadas seguintes os tratamentos não diferiram significativamente (Tabela 6).

Tabela 6. Estoque de Carbono (EC).

\begin{tabular}{ccccccc}
\hline \multirow{2}{*}{ Variável } & Prof. & \multicolumn{5}{c}{ Tratamentos* } \\
\cline { 3 - 7 } & $(\mathbf{c m})$ & ILPF-Paricá & PM & PE & FS & CV (\%) \\
\hline & $0-10$ & $18,79 \mathrm{Ba}$ & $18,24 \mathrm{Ba}$ & $31,61 \mathrm{Aa}$ & $28,74 \mathrm{Aa}$ & 9,19 \\
& $10-20$ & $18,23 \mathrm{ABa}$ & $17,80 \mathrm{Ba}$ & $17,36 \mathrm{Bb}$ & $20,69 \mathrm{Ab}$ & 8,00 \\
& $20-30$ & $11,73 \mathrm{Ab}$ & $13,55 \mathrm{Ab}$ & $12,62 \mathrm{Ac}$ & $12,45 \mathrm{Ac}$ & 14,03 \\
EC & $30-40$ & $8,44 \mathrm{Ac}$ & $9,41 \mathrm{Ac}$ & $9,19 \mathrm{Ad}$ & $9,11 \mathrm{Ad}$ & 11,87 \\
$\left(\mathbf{M g ~ h a}^{-1}\right)$ & $40-60$ & $7,94 \mathrm{Ad}$ & $7,72 \mathrm{Ad}$ & $7,82 \mathrm{Ade}$ & $7,95 \mathrm{Ae}$ & 5,86 \\
& $60-80$ & $7,82 \mathrm{Ad}$ & $7,65 \mathrm{Ad}$ & $7,66 \mathrm{Ade}$ & $7,81 \mathrm{Ae}$ & 12,87 \\
& $80-100$ & $5,06 \mathrm{Ae}$ & $6,86 \mathrm{Ae}$ & $6,86 \mathrm{Af}$ & $5,03 \mathrm{Af}$ & 12,18 \\
& $C V(\%)^{1}$ & 13,17 & 7,74 & 7,55 & 11,76 & - \\
\hline
\end{tabular}

*Médias seguidas da mesma letra maiúscula na linha e minúscula na coluna não diferem entre si pelo teste de Tukey $(p<0,05)$.

${ }^{1} \mathrm{CV}(\%)$ coeficiente de variação referente aos tratamentos (parcelas).

${ }^{2} \mathrm{CV}(\%)$ coeficiente de variação referente às camadas (subparcelas).

Estes resultados corroboram com os observados por Hickmann e Costa (2012), avaliando EC sob sistemas convencionais e conservacionistas, onde relataram redução de EC em profundidade. Resultados confirmados por Rossetti e Centurion (2015), ao avaliarem o estoque de carbono em cronossequência, indicaram declínio no EC na medida em que aumentou-se a profundidade.

De modo geral, os resultados de EC indicaram que as maiores diferenças e os maiores valores foram encontrados apenas nas camadas superficiais (Tabela 6), onde ocorre maior incremento de matéria orgânica. Resultados similares foram obtidos por Deon (2013), Durigan (2013) e Chaves (2014) que também realizaram estudos sobre estoque de carbono em Latossolo Amarelo no Bioma Amazônia.

Cerri et al. (2007), Tognon et al. (1998) e Camargo et al. (1999) declararam que a maior incorporação de matéria orgânica existente nos solos da Amazônia está contida em até $20 \mathrm{~cm}$ de profundidade, horizonte superficial do solo, justificado por ser o intervalo onde as alterações edáficas são mais aceleradas e perceptíveis em menor tempo, em razão ao maior acúmulo de COS e à atividade de microorganismos.
O baixo EC observado na camada $0-10 \mathrm{~cm}$ no ILPF-Paricá em relação à FS pode estar relacionado com sua recente implantação em área com histórico recente de pastagem degradada, conforme foi supracitada na avaliação do COS.

Valores similares de EC no tratamento PE quando comparado ao $\mathrm{FS}$, pode ser explicado pela presença das gramíneas no PE que exibem efeito rizosférico intenso por causa do seu alto volume radicular, que por meio da sua decomposição liberam nutrientes e também atua na formação de matéria orgânica do solo, favorecendo assim sua agregação (CAMPOS et al., 2016).

Constatou-se que os tratamentos ILPFParicá, PM e PE não foram capazes de recuperar os EC em todas as profundidades em comparação ao FS, fato que corrobora com dados de outros autores (CARVALHO et al., 2010; SALTON et al., 2014).

O carbono orgânico é considerado agente de formação e estabilização das partículas do solo (COGO et al., 2013), e a motivação do seu acúmulo deve ser prioridade, não apenas para o sequestro de carbono, mas também para melhorar qualidade, fertilidade, ciclagem de 
nutrientes e estruturação do solo (BARRETO et al., 2009; BRADY; WEIL, 2012).

Compreender a dinâmica do acúmulo de carbono orgânico no solo é importante para mensurar a sustentabilidade de agroecossistemas. A importância de identificar a contribuição dos diferentes arranjos produtivos de manejo do solo nos processos de formação e estocagem do carbono orgânico no solo e, por conseguinte, na ciclagem de nutrientes, consiste em determinar o potencial de cada sistema no sequestro de carbono e na capacidade de promover a melhoria da qualidade do solo e a possível contribuição para as mudanças climáticas (LAPOLA et al., 2014).

$\mathrm{Na}$ Região Amazônica, os sistemas de integração Lavoura-Pecuária-Floresta apresentam crescente adoção e os resultados mostram que a introdução de espécies florestais em consórcio com culturas anuais e forrageiras pode ser boa opção para aumentar a produtividade e, consequentemente, sequestrar carbono, melhorar a qualidade do solo e contribuir para a mitigação do aquecimento global em médio e longo prazo (THANGATA; HILDEBRAND, 2012; LOSS et al., 2014).

\section{CONCLUSÕES}

O sistema de integração LavouraPecuária-Floresta, aos cinco anos de cultivo, de modo geral, melhora as condições físicas de densidade e porosidade do solo, além dos teores e estoques de carbono orgânico nas camadas subsuperficiais, quando comparado aos sistemas convencionais e à floresta secundária.

O plantio convencional de milho promove aumento da densidade do solo e perda de porosidade total e microporosidade em profundidade. Entretanto, apresenta teores e estoques de carbono orgânicos similares ao sistema de integração Lavoura-Pecuária-Floresta.

A pastagem demonstra teores e estoques de carbono orgânico semelhante à floresta secundária, porém, indica redução de macroporosidade em todas as profundidades.

\section{REFERÊNCIAS}

ARAÚJO, A.S.F.; MONTEIRO, R.T.R. Indicadores biológicos de qualidade do solo. Bioscience Journal, v.23, n.3, p.66-75, 2007.

ARRUDA, E.M.; ALMEIDA, R.F.; SILVA JUNIOR, A.C.; RIBEIRO, B.T.; SILVA, A.A.; LANA, R.M.Q. Aggregation and organic matter content in different tillage systems for sugarcane. African Journal of Agricultural Research, v.10, n.4, p.281288, 2015. https://doi.org/10.5897/AJAR2014.9259.

BARRETO, R.C.; MADARI, B.E.; MADDOCK, J.E.L.; MACHADO, P.L.O.A.; TORRES, E.; FRANCHINI, J.; COSTA, A.R. The impact of soil management on aggregation, carbon stabilization and carbon loss as $\mathrm{CO} 2$ in the surface layer of a Rhodic Ferralsol in Southern Brazil. Agriculture, Ecosystems \& Environment, v.132, n.3, p.243-251, 2009. https://doi.org/10.1016/i.agee.2009.04.008.

BARTZ, M.L.C.; BROWN, G.G.; ROSA, M.G.; KLAUBERG, F.S.; JAMES, W.S.; DECAENS, T.; BARRETTA, D. Earthworm richness in land-use systems in Santa Catarina, Brazil. Applied Soil Ecology, v.83, p.59-70, 2014. https://doi.org/10.1016/i.apsoil.2014.03.003.

BAVER, L.D.; GARDNER, W.H.; GARDNER, W.R. Soil physics. 4. ed. New York: John Wiley and Sons, $1972.529 \mathrm{p}$.

BAYER, L.B.; BATJES, N.H.; BINDRABAN, P.S. Changes in organic carbon stocks upon land use conversion in the Brazilian Cerrado: a review. Agriculture, Ecosystems and Environment, v.137, p.47-58, 2010. https://doi.org/10.1016/j.agee.2010.02.003.

BETIOLI JUNIOR, E.; MOREIRA, W.H.; TORMENA, C.A.; FERREIRA, C.J.B.; SILVA, Á.P.; GIAROLA, N.F.B. Intervalo hídrico ótimo e grau de compactação de um latossolo vermelho após 30 anos sob plantio direto. Revista Brasileira de Ciência do Solo, v.36, n.3, 2012. http://dx.doi.org/10.1590/S010006832012000300027.

BRADY, N.C.; WEIL, R.R. Elementos da natureza e propriedades do solo. 3. ed. Porto Alegre: Bookman, 2012. p.55-97.

CAMARGO, P.B.; TRUMBORE, S.E.; MARTINELLI, L.A.; DAVIDSON, E.A.; NEPSTAD, D.C.; VICTORIA, R.L. Soil carbon dynamics in regrowing forest of eastern Amazonia. Global Change Biology, v.9, p.693-702, 1999 https://doi.org/10.1046/j.13652486.1999.00259.x.

CAMPOS, M.C.C.; SOARES, M.D.R.; NASCIMENTO, M.F.; SILVA, D.M.P. Estoque de carbono no solo e 
agregados em Cambissolo sob diferentes manejos no sul do Amazonas. Revista Ambiente \& Água, v.11, n.2, 2016. https://doi.org/10.4136/ambi-agua.1819.

CARVALHO, J.L.N.; CERRI, C.E.P.; FEIGEL, B.J.; PICCOLO, M.C.; GODINHO, V.P.; CERRI, C.C. Carbon sequestration in agricultural soils in the Cerrado region of the Brazil Amazon. Soil and Tillage Research, v.103, p.342-349, 2009. https://doi.org/10.1016/j.still.2008.10.022.

CARVALHO, J.L.N.; RAUCCI, G.S.; CERRI, C.E.P.; BERBOUX, M.; FEIGL, B.J.; WRUCK, F.J.; CERRI, C.C. Impact of pasture, agriculture and croplivestock systems on soil $C$ stocks in Brazil. Soil and Tillage Research, v.110, n.1, p.175-186, 2010. https://doi.org/10.1016/j.still.2010.07.011.

CERRI, C.E.P.; EASTER, M.; PAUSTIAN, K.; KILLIAN, K.; COLEMAN, K.; BERNOUX, M.; FALLOON, P.; POWLSON, D.S.; BATJES, N.H.; MILNE, E.; CERRI, C.C. Predicted soil organic carbon stocks and changes in the Brazilian Amazon between 2000 and 2030. Agriculture, Ecosystems and Environment, v.122, n.1, p.58-72, 2007. ttps://doi.org/10.1016/i.agee.2007.01.008.

CHAVES, S.S.F. Dinâmica do carbono no solo sob diferentes usos da terra em Paragominas, PA. 2014. 85p. Dissertação (Mestrado) - Escola Superior de Agricultura "Luiz de Queiroz", Universidade de São Paulo, Piracicaba-SP, 2014.

COGO, F.D.; JUNIOR, C.F.A.; ZINN, Y.L.; JUNIOR, M.S.D.; ALCÂNTARA, E.N.; GUIMARÃES, P.T.G. Estoques de carbono orgânico do solo em cafezais sob diferentes sistemas de controle de plantas invasoras. Semina: Ciências Agrárias, v.34, n.3, p.1089-1098, 2013. https://doi.org/10.5433/1679-

0359.2013v34n3p1089-1098.

COSTA, C.D.O.; ALVES, M.C.; SOUSA, A.P. Movimento de água e porosidade dos solos de uma sub-bacia hidrográfica no Noroeste do Estado de São Paulo. Irriga, v.20, n.2, p.304-318, 2015.

https://doi.org/10.15809/irriga.2015v20n2p304.

COSTA JR, C.; PICCOLO, M.C.; SIQUEIRA NETO, M.; BERNOUX. M. Carbono em agregados do solo sob vegetação nativa, pastagem e sistemas agrícolas no bioma Cerrado. Revista Brasileira de Ciência do Solo, v.33, n.1, p.1-12, 2012. https://doi.org/10.1590/S0100$\underline{06832012000400025}$.

CRUZ, D.L.S.; VALE JÚNIOR, J.F.; CRUZ, P.L.S.; CRUZ, A.B.S.; NASCIMENTO, P.P.R.R. Atributos físico-hídricos de um Argissolo amarelo sob floresta e savana naturais convertidas para pastagem em Roraima. Revista Brasileira de Ciência do Solo, v.38, n.1, p.307-314, 2014. https://doi.org/10.1590/S010006832014000100031.

DEON, D.S. Mudança de uso da terra e impacto na matéria orgânica do solo em dois locais no Leste da Amazônia. 2013. 151p. Tese (Doutorado) - Escola Superior de Agricultura "Luiz de Queiroz", Universidade de São Paulo, Piracicaba-SP, 2013. https://doi.org/10.11606/T.11.2013.tde$\underline{03072013-084639}$

DOMINGUES, M.S.; BERMANN, C. O arco de desflorestamento na Amazônia: da pecuária à soja. Ambiente \& Sociedade, v.15, n.2, p.1 -22, $2012 . \quad$ https://doi.org/10.1590/S1414753X2012000200002.

DURIGAN, M.R. Mudanças nos estoques de carbono e nitrogênio do solo em função da conversão do uso da terra no Pará. 2013. 101p. Dissertação (Mestrado) - Escola Superior de Agricultura "Luiz de Queiroz", Universidade de São Paulo, Piracicaba-SP, 2013. https://doi.org/10.11606/D.11.2013.tde$\underline{\text { 03062013-093119 }}$

EMBRAPA. Sistema Brasileiro de Classificação de Solos. 3. ed. Brasília: Embrapa, 2013. 353p.

EMBRAPA. Manual de métodos de análises do solo. 3. ed. Rio de Janeiro: Ministério da Agricultura, Embrapa Solos, 2011. 230 p.

FERREIRA, D.F. Sisvar: a computer statistical analysis system. Ciência \& Agrotecnologia, v.35, n.6, p.1039-1042, 2011. https://doi.org/10.1590/S141370542011000600001.

GRECO, A.M.F.; MUNARETO, J.D.; POZZEBON, B.C.; MUNARETO, G.S.; GALON, L.; BEUTLER, A.N. Influência do manejo nas propriedades físicas do solo. Anais do Salão Internacional de Ensino, Pesquisa e Extensão, v. 4, n. 2, 2012. 
GUAN, F.; TANG, X.; FAN, S.; ZHAO, J.; PENG, C. Changes in soil carbon and nitrogen stocks followed the conversion from secondary forest to Chinese fir and Moso bamboo plantations. Catena, v.133, n.22, p.455-460, 2015. https://doi.org/10.1016/j.catena.2015.03.002.

GUARESCHI, R.F.; PEREIRA, M.G.; PERIN, A. Deposição de resíduos vegetais, matéria orgânica leve, estoques de carbono e nitrogênio e fósforo remanescente sob diferentes sistemas de manejo no Cerrado Goiano. Revista Brasileira de Ciência do Solo, v.36, n.3, p.909-920, 2012. https://doi.org/10.1590/S0100$\underline{06832012000300021 .}$.

HICKMANN, C.; COSTA, L.M. Estoque de carbono no solo e agregados em argissolo sob diferentes manejos de longa duração. Revista Brasileira de Engenharia Agrícola e Ambiental, v.16, p.10551061, 2012. https://doi.org/10.1590/S141543662012001000004.

IBGE. Disponível em: $<$ http://www.sidra.ibge.gov.br/>. Acesso em: 28 abr. 2016.

INMET. Estações e dados/ Estações automáticas gráficos. 2017. Disponível em: $<$ http://www.inmet.gov.br/portal/index.php?r=h ome/page\&page=rede estacoes auto graf $>$.

Acesso em: 02 fev. 2017.

IWATA, B.F.; LEITE, L.F.C.; ARAÚJO, A.S.F.A.; NUNES, L.A.P.L.; GEHRING, C.; CAMPOS, L. P. Sistemas agroflorestais e seus efeitos sobre os atributos químicos em Argissolo VermelhoAmarelo do Cerrado piauiense. Revista Brasileira de Engenharia Agrícola e Ambiental, v.16, n.7, p.731-738, 2013. https://doi.org/10.1590/S141543662012000700005.

KAISER, D.R. Universidade Federal de Santa Maria. Centro de Ciências Rurais. Fundamentos da ciência do solo. Aulas práticas. Santa Maria, 112 p. 2010.

KIEHL, E.J. Manual de edafologia. 1. ed. São Paulo: Agronômica Ceres, 1979. 262p.

LAL, R. Forest soils and carbon sequestration. Forest Ecology and Management, v.220, n.1-3, p.242-258,

2005.

https://doi.org/10.1016/i.foreco.2005.08.015.

LAPOLA, D.M.; MARTINELLI, L.A.; PERES, C.A.; OMETTO, J.P.H.B.; FERREIRA, M.E.; NOBRE, C.A.; AGUIAR, A.P.D.; BUSTAMANTE, M.M.C.; CARDOSO, M.F.; COSTA, M.H.; JOLY, C.A.; LEITE, C.C.; MOUTINHO, P.; SAMPAIO, G.; STRASSBURG, B.B.N.; VIEIRA, I.C.G. Pervasive transition of the Brazilian land-use system. Nature Climate Change, v.4, p.27-35, 2014. https://doi.org/10.1038/nclimate2056.

LINHARES, J.M.S.; BASTOS, W.R.; JUNIOR, R.F.S.; OLIVEIRA, L.C.S. Variabilidade de atributos físicoquímicos e dos estoques de carbono orgânico em Argissolo Vermelho sob sistemas agrofloretais no Assentamento Umari Sul do Amazonas. Revista Geográfica Acadêmica, v.10, n.1, 2016.

LOSS, A.; PEREIRA, M.G.; ANJOS, L.H.C.; GIACOMO, S.G.; PERIN, A. Agregação, carbono e nitrogênio em agregados do solo sob plantio direto com integração lavoura-pecuária. Pesquisa Agropecuária Brasileira, v.46, p.565-579, 2011. https://doi.org/10.1590/S0100204X2011001000022.

LOSS, A.; RIBEIRO, E.C; PEREIRA, M.G; COSTA, E.M. Atributos físicos e químicos do solo em sistemas de consórcio e sucessão de lavoura, pastagem e silvipastoril em Santa Teresa, ES. Bioscience Journal, v.30, n.5, p.1347-1357, 2014.

MEDEIROS, I.B.B.G. Emprego de geotêxteis nãotecidos na percolação em solo arenoso com disposição de esgoto tratado sob condições anaeróbias. 2016. 34f. Dissertação (Mestrado) Universidade Federal do Rio Grande do Norte. Centro de Tecnologia, Natal-RN, 2016.

OLIVEIRA, P.R.; CENTURION, J.F.; CENTURION, M.A.P.C.; ROSSETI, K.V.; FERRAUDO, A.S.; FRANCO, H.B.J.; PEREIRA, F.S.; BÁRBARO JÚNIOR, L.S. Qualidade estrutural de um latossolo vermelho submetido à compactação. Revista Brasileira de Ciência do Solo, v.37, n.3, p.604-612, $2013 . \quad$ https://doi.org/10.1590/\$0100$\underline{06832013000300006 .}$.

OLIVEIRA, T.S.; COSTA, L.M.; FIGUEIREDO, M.S.; REGAZZI, A.J. Efeitos dos ciclos de umedecimento e secagem sobre a estabilidade de agregados em água de quatro Latossolos Brasileiros. Revista 
Brasileira de Ciência do Solo, v.20, n.3, p.509-515, 1996.

REICHERT, J.M.; SUZUKI, L.E.A.S.; REINERT, D.J. Compactação do solo em sistemas agropecuários e florestais: Identificação, efeitos, limites críticos e mitigação. In: CERETTA, C.A.; SILVA, L.S.; REICHERT, J.M. (Eds.) Tópicos em ciência do solo. 5. ed. Viçosa: Sociedade Brasileira de Ciência do Solo, 2007, p.49-134. https://doi.org/10.1016/j.still.2008.07.002

REICHERT, J.M.; SUZUKI, L.E.A.S.; REINERTA, D.J.; HORNB, R.; HÅKANSSONC, I. Reference bulk density and critical degree-of-compactness for no-till crop production in subtropical highly weathered soils. Soil and Tillage Research, v.102, n.2, p.242-254, 2009. https://doi.org/10.1016/j.still.2008.07.002.

ROSSETTI, K.V.; CENTURION, J.F. Estoque de carbono e atributos físicos de um Latossolo em cronossequência sob diferentes manejos. Revista Brasileira de Engenharia Agrícola e Ambiental, v.19, n.3, p.252-258, 2015. https://doi.org/10.5039/agraria.v8i2a2545.

SALTON, J.C.; MERCANTE, F.M.; TOMAZI, M.; ZANATTA, J.A.; CONCENÇO, G.; SILVA, W.M.; RETORE, $M$. Integrated crop-livestock system in tropical Brazil: Toward a sustainable production system. Agriculture, Ecosystems \& Environment, v.190, p.70-79, 2014. https://doi.org/10.1016/i.agee.2013.09.023.

SANTOS, G.G.; MARCHÃO, R.L.; SILVA, E.M.; SILVEIRA, P.M. BECQUER, T. Qualidade Física Do Dolo Sob Sistemas De Integração LavouraPecuária. Revista Pesquisa Agropecuária Brasileira, V.46, N.10, p.1339-1348, 2011. https://doi.org/10.1590/S0100-

204X2011001000030.

SILVA, A.R.; SALES, A.; VELOSO, C.A.C. Atributos físicos e disponibilidade de carbono do solo em sistemas de integração Lavoura-Pecuária-Floresta (ILPF), Homogêneo e Santa Fé, no estado do Pará, Brasil. Revista Agrotec, v.37, n.1, p.96-104, 2016. https://doi.org/10.1590/1807-

1929/agriambi.v19n4p309-316.

SILVA, E.F.; LOURENTE, E.P.R.; MARCHETTI, M.E.; MERCANTE, F.M.; FERREIRA, A.K.T.; FUJII, G.C. Frações lábeis e recalcitrantes da matéria orgânica em solos sob integração lavourapecuária. Pesquisa Agropecuária Brasileira, v.46, n.10, p.1321-1331, 2011.

https://doi.org/10.1590/S0100204X2011001000028.

SILVA, G.N. Sistemas de manejo e uso do solo sobre as frações e estoques de carbono e nitrogênio em Latossolo sob cerrado. 2016. 44p. Tese (Doutorado) - Universidade Federal de Lavras, Lavras-MG, 2016.

SOARES, M.D.R.; CAMPOS, M.C.C.; OLIVEIRA, I.A.; CUNHA, J.M.; SANTOS, L.A.C.; FONSECA, J.S.; SOUZA, Z.M.; Atributos físicos do solo em áreas sob diferentes sistemas de usos na região de Manicoré, AM. Revista de Ciências Agrárias, v.59, n.1, p.9-15, 2016. https://doi.org/10.4322/rca.2020.

SOUZA, E.D.; COSTA, S.E.V.G.A.; ANGHINONI, I.; CARVALHO, P.C.F.; ANDRIGUETI, M.; CAO, E. Estoque de carbono e nitrogênio total e nas frações da matéria orgânica do solo em sistema de integração agricultura-pecuária em plantio direto, submetido a intensidades de pastejo. Revista Brasileira de Ciência do Solo, v.33, n.6, p.1829-1836, 2009.

https://doi.org/10.1590/S0100$\underline{06832009000600031}$.

STEFANOSKI, D.C.; SANTOS, G.G.; MARCHÃO, R.L.; PETTER, F.A.; PACHECO, L.P. Uso e manejo do solo e seus impactos sobre a qualidade física. Revista Brasileira de Engenharia Agrícola e Ambiental, v.17, n.12, p.1301-1309, 2013. https://doi.org/10.1590/S1415$\underline{43662013001200008}$

THANGATA, P.H.; HILDEBRAND, P.E. Carbon stock and sequestration potential of agroforestry systems in smallholder agroecosystems of subSaharan Africa: Mechanisms for "reducing emissions from deforestation and forest degradation" (REDD+). Agriculture, Ecosystems \& Environment, v.158, n.1, p.172-183, 2012. https://doi.org/10.1016/j.agee.2012.06.007.

TOGNON, A.A.; DEMATTÊ, J.L.I.; DEMATTÊ, J.A.M. Teor e distribuição da matéria orgânica em latossolos das regiões da floresta amazônica e dos cerrados do Brasil Central. Scientia Agricola, v.55, p.343-354, 1998. 
https://doi.org/10.1590/S0103-

$\underline{90161998000300001 .}$.

TORRES, J.L.R.; SOUZA, Z.M.; PEREIRA, M.G.; ASSIS, R.L. Atributos indicadores da qualidade do solo numa área sob plantio direto há doze anos. Comunicata Scientiae, v.6, n.2, p.123-133, 2015.

VENDRUSCOLO, J.; RIBEIRO, T.S.; MESQUITA, F.O.; ALVES, A.S.; SANTOS, G.A. Propriedades físicas de um Latossolo e Argissolo comparados a quatro tipos de solos na Paraíba - Brasil. Revista Verde, v.6, n.4, p.204-212, 2011.

VEZZANI, F.M.; MIELNICZUK, J. Agregação e estoque de carbono em Argissolo submetido a diferentes práticas de manejo agrícola. Revista Brasileira de Ciência do Solo, v.35, p.213-223, $2011 . \quad$ https://doi.org/10.1590/S010006832011000100020.

Recebido para publicação em 05/09/2017

Revisado em 28/01/2018

Aceito em 24/03/2018 